\title{
Article
}

\section{Weak Association between Skin Autofluorescence Levels and Prediabetes with an ILERVAS Cross-Sectional Study}

\author{
Enric Sánchez ${ }^{1} \oplus$, Mohsen Kerkeni ${ }^{2}{ }^{\circledR}$, Marta Hernández ${ }^{1}$, Ricard Gavaldà ${ }^{3}$, Ferran Rius ${ }^{1}$, Ariadna Sauret ${ }^{1}$, \\ Gerard Torres ${ }^{4}$, Marcelino Bermúdez-López ${ }^{5,6}$, Elvira Fernández ${ }^{5,6}$, Eva Castro-Boqué ${ }^{5,6}$, Francisco Purroy ${ }^{7}$, \\ Dídac Mauricio ${ }^{8,9}{ }^{\mathbb{D}}$, Cristina Farràs-Sallés ${ }^{10}$, Miquel Buti ${ }^{10}$, Pere Godoy ${ }^{7}$, Reinald Pamplona ${ }^{11} \mathbb{C}$ \\ and Albert Lecube ${ }^{1,9, *,+}$ on behalf of the ILERVAS Project Collaborators
}

Citation: Sánchez, E.; Kerkeni, M.; Hernández, M.; Gavaldà, R.; Rius, F.;

Sauret, A.; Torres, G.

Bermúdez-López, M.; Fernández, E.; Castro-Boqué, E.; et al. Weak Association between Skin

Autofluorescence Levels and

Prediabetes with an ILERVAS

Cross-Sectional Study. Nutrients 2022, 14, 1102. https://doi.org/10.3390/ nu14051102

Academic Editor: Leni Rose Rivera

Received: 28 January 2022

Accepted: 3 March 2022

Published: 5 March 2022

Publisher's Note: MDPI stays neutral with regard to jurisdictional claims in published maps and institutional affiliations.

Copyright: (C) 2022 by the authors. Licensee MDPI, Basel, Switzerland. This article is an open access article distributed under the terms and conditions of the Creative Commons Attribution (CC BY) license (https:// creativecommons.org/licenses/by/ $4.0 /)$.
1 Endocrinology and Nutrition Department, Diabetes and Metabolism (ODIM) Research Group, University Hospital Arnau de Vilanova, Obesity, IRBLleida, University of Lleida, 25198 Lleida, Spain; esanchez@irblleida.cat (E.S.); mhernandezg.lleida.ics@gencat.cat (M.H.); frius.lleida.ics@gencat.cat (F.R.); ariadnags973@gmail.com (A.S.)

2 Laboratory of Research on Biologically Compatible Compounds, Faculty of Dental Medicine, University of Monastir, Monastir 5000, Tunisia; mohsen.kerkeni@yahoo.fr

3 Amalfi Analytics, Polytechnic University of Catalonia, 08034 Barcelona, Spain; rgavalda@irblleida.cat

4 Precision Medicine in Chronic Diseases Group, IRBLleida, 25198 Lleida, Spain; gtorres@gss.scs.es

5 Vascular and Renal Translational Research Group, IRBLleida, 25198 Lleida, Spain; mbermudez@irblleida.cat (M.B.-L.); efernandez@irblleida.cat (E.F.); ecastro@irblleida.cat (E.C.-B.)

6 Red de Investigación Renal, Instituto de Salud Carlos III (RedinRen-ISCIII), 28029 Madrid, Spain

7 Stroke Unit, Clinical Neurosciences Group, University Hospital Arnau de Vilanova, IRBLleida, University of Lleida, 25198 Lleida, Spain; fpurroygarcia@gmail.com (F.P.); pere.godoy@gencat.cat (P.G.)

8 Endocrinology and Nutrition Department, Hospital de la Santa Creu i Sant Pau, Institut de Recerca Biomèdica Sant Pau (IIB Sant Pau), 08025 Barcelona, Spain; didacmauricio@gmail.com

9 Centro de Investigación Biomédica en Red de Diabetes y Enfermedades Metabólicas Asociadas (CIBERDEM), Instituto de Salud Carlos III (ISCIII), 28029 Madrid, Spain

10 Applied Epidemiology Research Group, IRBLleida. Unitat de Suport a la Recerca Lleida, Fundació Institut Universitari per a la Recerca a l'Atenció Primària de Salut Jordi Gol i Gurina (IDIAPJGol), 25198 Lleida, Spain cfarras.lleida.ics@gencat.cat (C.F.-S.); mbuti.lleida.ics@gencat.cat (M.B.)

11 Department of Experimental Medicine, IRBLleida, University of Lleida, 25198 Lleida, Spain; reinald.pamplona@mex.udl.cat

* Correspondence: alecube.1leida.ics@gencat.cat; Tel.: +34-973-70-51-83; Fax: +34-973-70-51-89

+ Collaborators of the ILERVAS project are indicated in the Acknowledgment section.

Abstract: A large body of evidence demonstrates a relationship between hyperglycemia and increased concentrations of advanced glycation end-products (AGEs). However, there is little information about subcutaneous AGE accumulation in subjects with prediabetes, and whether or not this measurement could assist in the diagnosis of prediabetes is unclear. A cross-sectional study was conducted in 4181 middle-aged subjects without diabetes. Prediabetes $(n=1444)$ was defined as a glycosylated hemoglobin (HbA1c) level between 39 and $47 \mathrm{mmol} / \mathrm{mol}$ (5.7 to $6.4 \%$ ), and skin autofluorescence (SAF) measurement was performed to assess AGEs. A multivariable logistic regression model and receiver operating characteristic curve were used. The cohort consisted of $50.1 \%$ women with an age of 57 [52;62] years, a BMI of $28.3[25.4 ; 31.6] \mathrm{kg} / \mathrm{m}^{2}$, and a prevalence of prediabetes of $34.5 \%$. Participants with prediabetes showed higher SAF than control participants $(2.0$ [1.7;2.2] vs. $1.9[1.7 ; 2.2], p<0.001)$. However, HbA1c was not significantly correlated with SAF levels $(\mathrm{r}=0.026$, $p=0.090)$. In addition, the SAF level was not independently associated with prediabetes (OR $=1.12$ (0.96 to 1.30)). Finally, there was no good cutoff point for SAF to identify patients with prediabetes (AUC $=0.52(0.50$ to 0.54$)$, sensitivity $=0.61$, and 1-specificity $=0.56)$. Given all of this evidence, we can conclude that although there is an increase in SAF levels in participants with prediabetes, the applicability and clinical relevance of the results is low in this population.

Keywords: advanced glycation end-products; glycosylated hemoglobin; prediabetes; skin autofluorescence 


\section{Introduction}

Advanced glycation end-products (AGEs) constitute a complex group of compounds formed by the slow non-enzymatic glycation of proteins, lipids, and nucleic acids, of which about 20 have been identified to date [1]. AGEs trigger the formation of cross-links between extracellular matrix basement membrane molecules and the activation of receptors for AGEs. These pathological mechanisms contribute to, among other issues, endothelial dysfunction, formation of reactive oxygen species, and vascular stiffness [2]. Aging leads to an increase in the concentration of AGEs in tissues and the circulation, which is accelerated by chronic hyperglycemia and smoking, resulting in increased oxidative stress and cellular damage [3,4]. New studies have suggested that the abundance of prooxidant AGEs in the highly industrialized food environment may explain the onset and progression of prediabetes to type 2 diabetes [5].

Prediabetes, an intermediate metabolic state between type 2 diabetes and normal glucose metabolism, showed a prevalence of $38.6 \%$ based on fasting plasma glucose (FPG)/hemoglobin A1c (HbA1c) in the adult population according to the National Health and Nutrition Examination Survey (NHANES) performed between 2017 and 2020 [6]. It is essential to note that prediabetes is not an inconsequential health condition, as it has been associated with an increased incidence of cardiovascular disease and diabetic microangiopathy compared with the population with normal glucose metabolism [7-9]. Subcutaneous AGE content, assessed by skin autofluorescence (SAF), has been implicated in the early development of both plaque-burden-associated risk factors and classic microangiopathic complications in diabetes [10-12]

It is well known that the main challenge in the prevention of type 2 diabetes is the early identification of individuals with prediabetes to introduce actions to postpone the onset of future disease [13]. However, the best way to perform this screening has yet to be decided. Thus, measures such as FPG, $2 \mathrm{~h}$ plasma glucose, and $\mathrm{HbA1c}$ are equally suitable for identifying prediabetes and diabetes, but not all of them identify the disease in the same individual [14]. The measurement of AGEs seems to be a suitable way to assess the effects of years of chronic non-diabetic hyperglycemia, and subcutaneous AGE deposition has been previously evaluated in populations at risk for diabetes with positive results [15-20]. However, it remains to be elucidated whether the measurement of AGE deposition in subcutaneous tissue can help to differentiate subjects with prediabetes from those with normal glucose metabolism. Therefore, the aim of our study was to evaluate the concentration of subcutaneous AGE in prediabetes, as well as determine whether its measurement could be a good option for the diagnosis of this metabolic state, by examining a population with low-to-moderate cardiovascular risk from the ILERVAS study.

\section{Materials and Methods}

\subsection{Ethical Statement}

The protocol was accepted by the ethics committee of the University Hospital Arnau de Vilanova (CEIC-1410). The clinical trial was conducted in accordance with the ethical guidelines of the Declaration of Helsinki and Spanish legislation on personal data protection. All subjects provided written informed consent.

\subsection{Design of the Study and Data of the Study Participants}

The ILERVAS project is an ongoing randomized intervention study with the aim of reducing cardiovascular events in Catalonia, Spain (ClinTrials.gov Identifier: NCT03228459) [21]. Between January 2015 and December 2018, 8330 middle-aged participants were recruited from various primary care centers. Inclusion criteria were women aged 50 to 70 years, men aged 45 to 65 years, and the presence of at least one cardiovascular risk factor (dyslipidemia, hypertension, obesity, smoking, and/or having a first-degree relative with premature cardiovascular disease). Exclusion criteria were a clinical history of cardiovascular disease, any type of diabetes, renal disease, active neoplasia, a life expectancy of less than 1 year, institutionalized population, and pregnancy. The study flow chart is displayed in Figure 1. 
8330 patients were recruited between January 2015 and December 2018

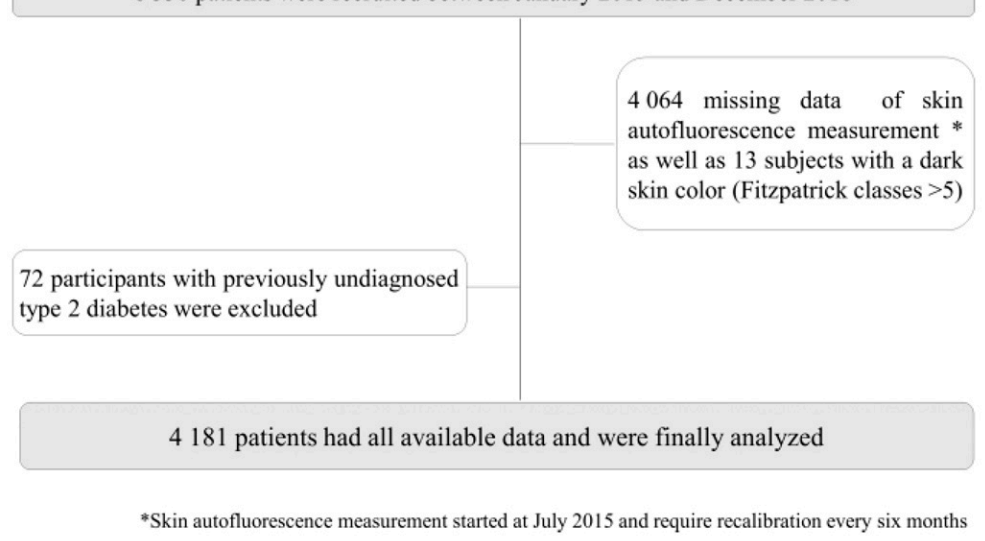

Figure 1. Flow diagram for the study population.

\subsection{Measurement of Skin Autofluorescence}

SAF was calculated using the AGE Reader ${ }^{\mathrm{TM}}$ (DiagnOptics Technologies, Groningen, The Netherlands), a fully automated non-invasive tool that assesses AGE deposition on the forearm using ultraviolet A light [22]. The mean value of three analyses (arbitrary units: AUs) was recorded. Areas of tattooed or cosmetically colored skin and areas with many freckles or vessels close to the skin surface were avoided. A single device, maintained and calibrated by the manufacturer according to their recommendations, was used for all measurements. Repeated SAF measurements with the AGE Reader ${ }^{\mathrm{TM}}$ device taken on a single day showed an overall Altman error rate of $5.03 \%$, and intra-individual seasonal variance showed an Altman error rate of 5.87\% [22].

\subsection{Diagnosis of Prediabetes and Type 2 Diabetes}

The current American Diabetes Association guidelines define type 2 diabetes as $\mathrm{HbA1c} \geq 48 \mathrm{mmol} / \mathrm{mol}(\geq 6.5 \%)$, prediabetes as $\mathrm{HbA1c}$ between 39 and $47 \mathrm{mmol} / \mathrm{mol}$ (5.7 to $6.4 \%$ ), and normal glucose metabolism as $\mathrm{HbA1c}<39 \mathrm{mmol} / \mathrm{mol}(<5.7 \%)$ [14]. A total of 72 participants with previously undiagnosed type 2 diabetes were excluded from the analysis, which was finally performed in 4181 individuals. The $\mathrm{HbA1c}$ test was performed on capillary blood using a point-of-care device (Cobas B $101^{\circledR}$, Roche Diagnostics S.L., Sant Cugat del Vallès, Spain) based on a latex agglutination inhibition immunoassay technique that meets generally accepted performance standards for $\mathrm{HbA1c}$. Subjects with prediabetes were divided into those with low (5.7\% to $6.0 \%)$ and high $(6.1 \%$ to $6.4 \%) \mathrm{HbA} 1 \mathrm{c}$ due to the non-normal distribution of the variable. Body weight and height were measured without shoes and with little clothing, and body mass index (BMI) was determined by dividing weight in kilograms by height in meters squared. Smoking status (never, former, or current smoker) was also obtained. Smokers who quit smoking one year or more before the visit were considered ex-smokers.

\subsection{Statistical Analysis}

The normal distribution of the variables was assessed using the Shapiro-Wilk test, and the data are expressed as median (interquartile range) or as a percentage. The MannWhitney U test was used to compare continuous variables, while Pearson's chi-square test was used to compare categorical data. The relationship between continuous variables was examined using Spearman's correlation test.

Multivariable logistic regression was performed to identify variables that are independently related to prediabetes. Variables with a potential impact on the measurement of prediabetes (i.e., age, sex, BMI, and smoking) and the levels of SAF were entered into the model. Additionally, the multivariable logistic regression model was calibrated by both the Hosmer-Lemeshow test of fit and area under the ROC curve. 
Finally, the area under the receiver operating characteristic (ROC) curve showing sensitivity/specificity was calculated to examine the diagnostic ability of skin autofluorescence to discriminate prediabetes. The total area under the ROC curve was interpreted using the following guidelines: 0.9-1.0, excellent; 0.8-0.9, good; 0.7-0.8, fair; 0.6-0.7, poor; and $0.5-0.6$, not useful. All $p$-values were based on a two-sided statistical significance test. Significance was set at the level of $p<0.050$. All statistical investigations were performed with the SSPS statistical package (IBM SPSS Statistics for Windows, version 27.0. Armonk, NY, USA).

\section{Results}

The main clinical characteristics of the ILERVAS population according to the presence or absence of prediabetes are shown in Table 1. Subjects with prediabetes were older, mainly women, and had a higher proportion of non-communicable diseases than controls. Subjects with prediabetes presenting high $\mathrm{HbA1c}$ values $(6.1 \%$ to $6.4 \%)$ appeared to be under-represented $(17.5 \%)$.

Table 1. Main clinical characteristics of the study population according to glucose abnormalities.

\begin{tabular}{ccc}
\hline & Prediabetes $(\boldsymbol{n}=\mathbf{1 4 4 4})$ & Control Group $(\boldsymbol{n}=\mathbf{2 7 3 7})$ \\
\hline Age $($ years $)$ & $59[54 ; 64]$ & $56[52 ; 62]$ \\
Women, $n(\%)$ & $827(57.3)$ & $1268(46.3)$ \\
BMI $\left(\mathrm{kg} / \mathrm{m}^{2}\right)$ & $29.5[26.7 ; 33.1]$ & $27.7[24.8 ; 30.8]$ \\
HbA1c $(\mathrm{mmol} / \mathrm{mol})$ & $40[39 ; 42]$ & $36[33 ; 37]$ \\
HbA1c $(\%)$ & $5.8[5.7 ; 6.0]$ & $5.4[5.2 ; 5.5]$ \\
Hypertension, $n(\%)$ & $684(47.4)$ & $992(36.2)$ \\
Dyslipidemia & $865(59.9)$ & $1363(49.8)$ \\
Obesity, $n(\%)$ & $581(40.2)$ & $758(27.7)$ \\
Current or former smoker, $n(\%)$ & $828(57.3)$ & $1819(66.5)$ \\
\hline
\end{tabular}

Data are presented as a median [interquartile range] or $n$ (percentage). HbA1c: glycated hemoglobin; BMI: body mass index.

Participants with prediabetes showed small but statistically significant increases in SAF compared to controls $(2.0$ [1.7;2.2] vs. 1.9 [1.7;2.2], $p<0.001)$ (Figure 2). Furthermore, when this comparison was made in patients with prediabetes, similar results were obtained when comparing those with higher $\mathrm{HbA} 1 \mathrm{c}$ levels $(6.1 \%$ to $6.4 \%)$ with those with a lower HbA1c concentration for SAF levels: 2.0 [1.7;2.2] vs. 1.9 [1.7;2.2], $p<0.001$.

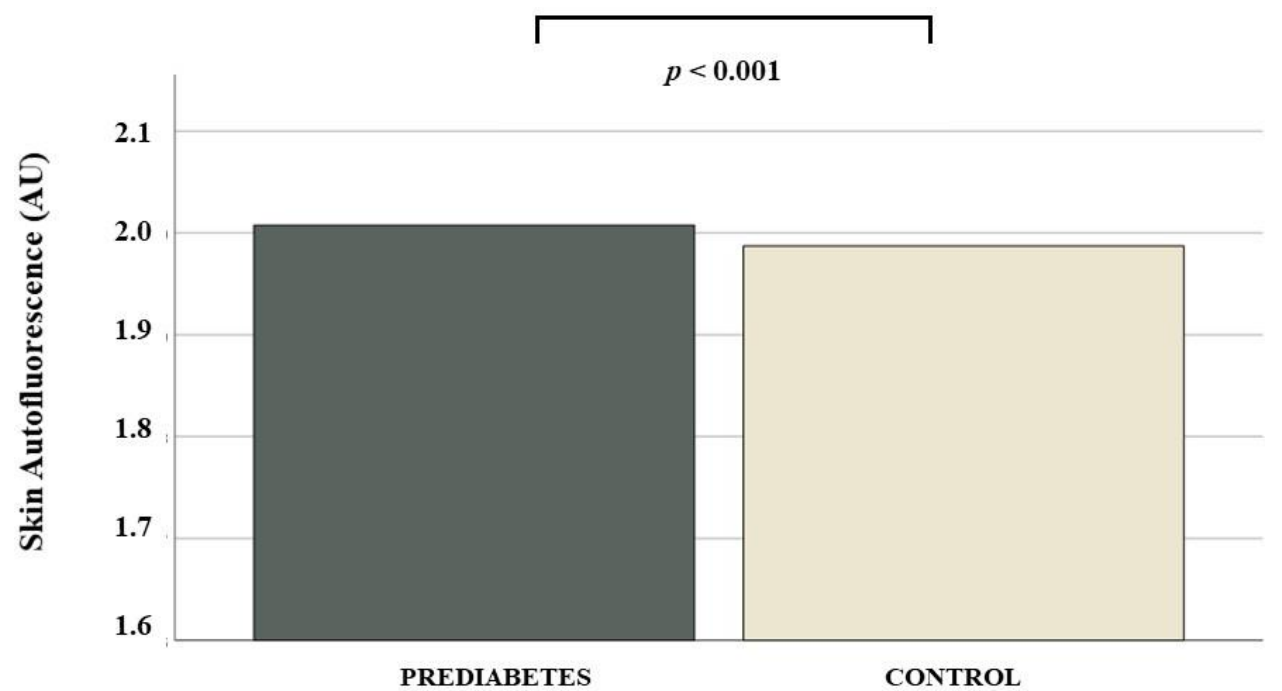

Figure 2. Plot displaying the skin autofluorescence value according to the presence prediabetes. 
According to the quartiles of SAF levels, the prevalence of prediabetes increased significantly $(31.0 \%, 35.7 \%, 37.9 \%$, and $34.8 \%$, respectively, $p=0.006)$. Similar results were observed when $\mathrm{HbA} 1 \mathrm{c}$ was evaluated according to SAF quartiles $(p=0.005)$. In the whole population, the SAF level was not significantly correlated with $\mathrm{HbA1c}(\mathrm{r}=0.026, p=0.090)$.

Furthermore, the multivariable logistic regression model (Table 2) showed that male sex and smoking, but not the SAF level, were significantly and independently associated with prediabetes. The results of this model were similar after stratifying for obesity, hypertension, and dyslipidemia.

Table 2. The multivariable logistic regression model for presence of prediabetes.

\begin{tabular}{|c|c|c|c|}
\hline \multicolumn{2}{|c|}{ Prediabetes } & \multirow{2}{*}{$\begin{array}{c}\text { OR }(95 \% \text { CI) } \\
\text { Reference }\end{array}$} & \multirow[t]{2}{*}{$p$ Value } \\
\hline$C_{1}$ & Women & & \\
\hline sex & Men & 1.34 (1.16 to 1.55$)$ & $<0.001$ \\
\hline \multicolumn{2}{|c|}{ Age (years) } & 1.04 (1.03 to 1.05$)$ & $<0.001$ \\
\hline \multicolumn{2}{|c|}{ Body mass index $\left(\mathrm{kg} / \mathrm{m}^{2}\right)$} & 1.09 (1.07 to 1.10$)$ & $<0.001$ \\
\hline \multirow{2}{*}{ Smoking status } & Never & Reference & \\
\hline & Current or former & 1.08 (0.93 to 1.25$)$ & 0.326 \\
\hline \multicolumn{2}{|c|}{ Skin autofluorescence (AU) } & $0.97(1.00$ to 0.85$)$ & 0.969 \\
\hline \multicolumn{2}{|c|}{$\begin{array}{l}\text { Hosmer-Lemeshow test of fit } \\
\text { Area under the ROC curve }\end{array}$} & 0.65 (0.64 to 0.67$)$ & $\begin{array}{c}0.002 \\
<0.001\end{array}$ \\
\hline
\end{tabular}

Finally, ROC analysis revealed that SAF measurement was not useful (considering both sensitivity and specificity) for identifying patients with prediabetes in our population $(\mathrm{AUC}=0.52(0.50$ to 0.54$)$, sensitivity $=0.61$, and 1-specificity $=0.56)($ Figure 3$)$.

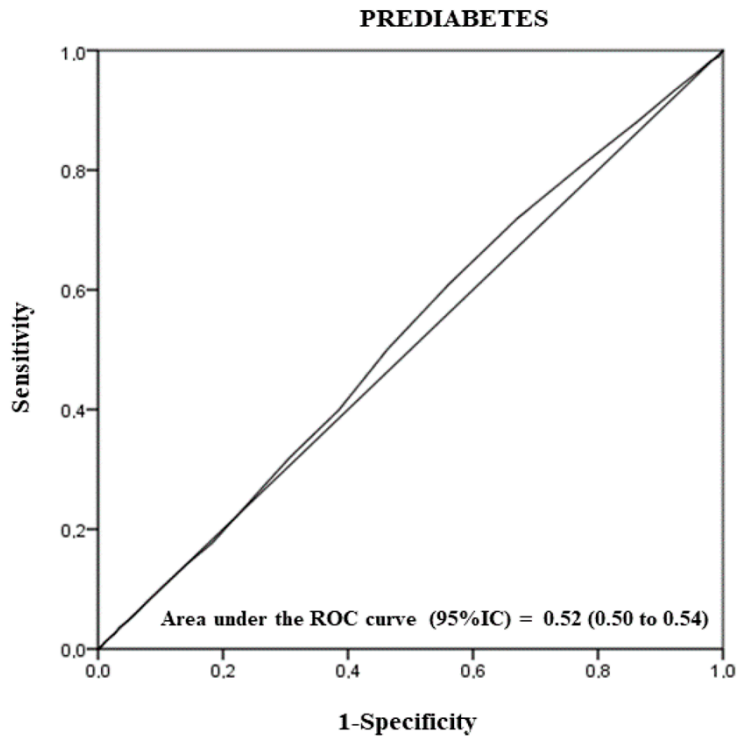

Figure 3. Receiver operating characteristic (ROC) curve analysis to evaluate the accuracy of skin autofluorescence in distinguishing prediabetes from cases with normal metabolism, together with sensitivity/specificity data.

\section{Discussion}

In the present study, we provide evidence that, although the prediabetes stage is associated with a modest increase in SAF, its measurement does not appear to be useful for screening for prediabetes, at least not in the population with low-to-moderate cardiovascular risk from the ILERVAS study. The period of mild hyperglycemia preceding the development of type 2 diabetes exerts a negative effect on health, facilitating the development of various risk factors associated with cardiovascular disease and classic microangiopathic complications [8,9]. The role of AGEs in the etiopathogenesis of late complications in type 2 diabetes is well known. In addition, a recent study suggested that SAF is 
associated with vascular stiffness in prediabetes and normoglycemia in a population-based cohort without high CV disease risk [23].

In recent years, much research has been conducted to explain the negative impact of AGEs on human health and to identify the pathological mechanisms involved in the development and progression of type 2 diabetes, such as insulin resistance and cellular dysfunction [24]. The association of AGEs with HbA1c and chronic complications of type 2 diabetes has also been described, suggesting that the measurement of AGEs could be a potential biomarker to predict progression from normal glucose metabolism to type 2 diabetes [25-28]. For this reason, the measurement of SAF, using increasingly smaller, more manageable, and less expensive tabletop devices, is becoming more widespread in the assessment of cardiovascular risk [29]. It could also offer an opportunity for prediabetes screening in populations with varying degrees of CV risk. However, our study failed to establish a correlation between SAF and metabolic control in the prediabetes phase. We did find a higher concentration of AGEs in subjects with prediabetes compared to those with normal glucose metabolism. However, although this difference was statistically significant, probably due to the large population evaluated, it does not appear to be clinically relevant.

Among individuals at high risk for type 2 diabetes, SAF has previously been shown to have equal or superior diagnostic performance for prediabetes compared to FPG and HbA1c [15-20,30]. In the study by Maynard et al. involving 351 subjects with one or more diabetes risk factors, the sensitivity differential indicated that SAF was able to identify $28.8 \%$ more individuals with 2 -h OGTT values $\geq 140 \mathrm{mg} / \mathrm{dL}$ than FPG testing and $17.1 \%$ more than $\mathrm{HbA1c}$ testing [16]. Similarly, in the study by Smit et al. in 218 subjects meeting at least one metabolic syndrome criterion, the performance of a SAF-based decision model was better than conventional FPG and comparable to $\mathrm{HbA} 1 \mathrm{c}$ in detecting impaired glucose tolerance or diabetes [15]. Furthermore, in both the NSEEDS and ENGINE studies, SAF was compared with FPG and $\mathrm{HbA1c}$ for the detection of abnormal glucose tolerance in subjects at risk for type 2 diabetes but without an established diagnosis [18,19]. Finally, $1 \mathrm{~h}$ post-glucose load plus SAF appeared to be the best combination in the assessment of prediabetes among the Diabetes Prediction and Screening Observational (DIAPASON) study cohort, which included subjects aged 40 to 75 years and a FINDRISC $\geq 9$, [17]. However, in our study, although we showed slightly elevated levels of SAF in the ILERVAS population when prediabetes was defined according to $\mathrm{HbA1c}$ criteria, we did not observe this clinical utility

This study has some limitations. First, it would have been interesting to compare the SAF data with plasma AGE concentrations. However, AGE deposition seems to be a more reliable measure of the negative impact of these compounds on human health since SAF remains stable for a long time due to its slow turnover. Second, our population had an $\mathrm{HbA} 1 \mathrm{c}$ level of $5.8 \%$, which is in the lower cutoff range for the diagnosis of prediabetes. This would suggest that subjects with higher $\mathrm{HbA1c}$ levels within the prediabetes range were under-represented. SAF is related to metabolic control about 10 years earlier and not to current metabolic control, which may explain why SAF shows a low discriminatory ability compared to $\mathrm{HbA1c}$, which captures more recent effects in its measurement [31]. Finally, in contrast to previous studies, we did not evaluate a population at high risk of developing diabetes but a population at low-to-moderate cardiovascular risk. This difference between study populations could lead to different results.

\section{Conclusions}

In sum, there is a modest increase in SAF levels in participants with prediabetes that is mainly associated with male sex and smoking. However, we did not observe a significant and independent association between the presence of prediabetes and high SAF levels. In addition, there is not a cutoff point for SAF that has both the sensitivity and specificity needed to identify patients with prediabetes. Therefore, SAF does not appear to be a good tool to identify subjects with prediabetes among the population with low-to-moderate moderate $\mathrm{CV}$ risk. 
Author Contributions: Conceptualization, E.S.; data curation, M.K., M.H. and R.G.; formal analysis, M.K., M.H. and R.G.; funding acquisition, M.B.-L. and E.F.; investigation, F.R., A.S. and G.T.; methodology, F.R., A.S. and G.T.; project administration, M.B.-L., E.F. and E.C.-B.; resources, M.B.-L., E.F. and E.C.-B.; supervision, F.P., D.M., C.F.-S., M.B., P.G. and R.P.; visualization, F.P., D.M., C.F.-S., M.B., P.G. and R.P.; writing-original draft, E.S. and A.L.; writing—review and editing, E.S. and A.L. All authors have read and agreed to the published version of the manuscript.

Funding: This work was supported by grants from the Diputació de Lleida and Generalitat de Catalunya (2017SGR696 and SLT0021600250). CIBER de Diabetes y Enfermedades Metabólicas Asociadas and CIBER de Enfermedades Respiratorias are initiatives of the Instituto de Salud Carlos III.

Institutional Review Board Statement: The study was conducted according to the guidelines of the Declaration of Helsinki and approved by Arnau de Vilanova University Hospital Ethics Committee (CEIC-1410, 19 December 2014).

Informed Consent Statement: Informed consent was obtained from all subjects involved in the study.

Data Availability Statement: The data presented in this study are available on request from the corresponding author. The data are not publicly available due to the signed consent agreements around data sharing, which only allow access to the researchers of the study following the project purposes. Requestors wishing to access the data used in this study can make a request to A.L. and M.B.-L. The request will be subjected to approval and formal agreements regarding confidentiality and secure data storage being signed the data would be the provided.

Acknowledgments: The authors would like to thank the staff of El Bus de la Salut, Fundació Renal Jaume Arnó, the primary care teams of the province of Lleida, and the collaborators of the ILERVAS project.

Conflicts of Interest: The authors declare no conflict of interest.

\section{References}

1. Ajith, T.A.; Vinodkumar, P. Advanced glycation end products: Association with the pathogenesis of diseases and the current therapeutic approaches. Curr. Clin. Pharmacol. 2016, 11, 118-127. [CrossRef] [PubMed]

2. Bierhaus, A.; Hofmann, M.A.; Ziegler, R.; Nawroth, P.P. AGEs and their interaction with AGE-receptors in vascular disease and diabetes mellitus. I. The AGE concept. Cardiovasc. Res. 1998, 37, 586-600. [CrossRef]

3. Goh, S.Y.; Cooper, M.E. The role of advanced glycation end products in progression and complications of diabetes. J. Clin. Endocrinol. Metab. 2008, 93, 1143-1152. [CrossRef] [PubMed]

4. Vlassara, H.; Striker, G.E. Advanced glycation endproducts in diabetes and diabetic complications. Endocrinol. Metab. Clin. N. Am. 2013, 42, 697-719. [CrossRef] [PubMed]

5. Uribarri, J.; Cai, W.; Peppa, M.; Goodman, S.; Ferrucci, L.; Striker, G.; Vlassara, H. Circulating glycotoxins and dietary advanced glycation endproducts: Two links to inflammatory response, oxidative stress, and aging. J. Gerontol. Ser. A Biol. Sci. Med. Sci. 2007, 62, 427-433. [CrossRef]

6. Xia, P.F.; Tian, Y.X.; Geng, T.T.; Li, Y.; Tu, Z.Z.; Zhang, Y.B.; Guo, K.; Yang, K.; Liu, G.; Pan, A. Trends in Prevalence and Awareness of Prediabetes Among Adults in the U.S., 2005-2020. Diabetes Care 2022, 45, e21-e23. [CrossRef]

7. Huang, Y.; Cai, X.; Mai, W.; Li, M.; Hu, Y. Association between prediabetes and risk of cardiovascular disease and all cause mortality: Systematic review and meta-analysis. BMJ 2016, 355, i5953. [CrossRef]

8. Brunner, E.J.; Shipley, M.J.; Witte, D.R.; Fuller, J.H.; Marmot, M.G. Relation between blood glucose and coronary mortality over 33 years in the Whitehall Study. Diabetes Care 2006, 29, 26-31. [CrossRef]

9. Sánchez, E.; Betriu, À.; López-Cano, C.; Hernández, M.; Fernández, E.; Purroy, F.; Bermúdez-López, M.; Farràs-Sallés, C.; Barril, S.; Pamplona, R.; et al. Characteristics of atheromatosis in the prediabetes stage: A cross-sectional investigation of the ILERVAS project. Cardiovasc. Diabetol. 2019, 18, 154. [CrossRef]

10. Goldin, A.; Beckman, J.A.; Schmidt, A.M.; Creager, M.A. Advanced glycation end products: Sparking the development of diabetic vascular injury. Circulation 2006, 114, 597-605. [CrossRef]

11. Kimura, H.; Tanaka, K.; Kanno, M.; Watanabe, K.; Hayashi, Y.; Asahi, K.; Suzuki, H.; Sato, K.; Sakaue, M.; Terawaki, H.; et al. Skin autofluorescence predicts cardiovascular mortality in patients on chronic hemodialysis. Ther. Apher. Dial. 2014, 18, $461-467$. [CrossRef] [PubMed]

12. Vélayoudom-Céphise, F.L.; Rajaobelina, K.; Helmer, C.; Nov, S.; Pupier, E.; Blanco, L.; Hugo, M.; Farges, B.; Astrugue, C.; Gin, H.; et al . Skin autofluorescence predicts cardio-renal outcome in type 1 diabetes: A longitudinal study. Cardiovasc. Diabetol. 2016, 15, 127. [CrossRef] [PubMed]

13. Esquivel Zuniga, R.; DeBoer, M.D. Prediabetes in Adolescents: Prevalence, Management and Diabetes Prevention Strategies. Diabetes Metab. Syndr. Obes. Targets Ther. 2021, 14, 4609-4619. [CrossRef] [PubMed] 
14. American Diabetes Association. Classification and diagnosis of diabetes. Section 2. Diabetes Care 2017, 40, S11-S24. [CrossRef] [PubMed]

15. Smit, A.J.; Smit, J.M.; Botterblom, G.J.; Mulder, D.J. Skin autofluorescence based decision tree in detection of impaired glucose tolerance and diabetes. PLoS ONE 2013, 8, e65592. [CrossRef] [PubMed]

16. Maynard, J.D.; Rohrscheib, M.; Way, J.F.; Nguyen, C.M.; Ediger, M.N. Noninvasive type 2 diabetes screening, superior sensitivity to fasting plasma glucose and A1C. Diabetes Care 2007, 30, 1120-1124. [CrossRef]

17. La Sala, L.; Tagliabue, E.; de Candia, P.; Prattichizzo, F.; Ceriello, A. One-hour plasma glucose combined with skin autofluorescence identifies subjects with pre-diabetes: The DIAPASON study. BMJ Open Diabetes Res. Care 2020, 8, e001331. [CrossRef]

18. Hull, E.L.; Matter, N.I.; Olson, B.P.; Ediger, M.N.; Magee, A.J.; Way, J.F.; Vugrin, K.E.; Maynard, J.D. Noninvasive skin fluorescence spectroscopy for detection of abnormal glucose tolerance. J. Clin. Transl. Endocrinol. 2014, 1, 92-99. [CrossRef]

19. Stirban, A. Noninvasive skin fluorescence spectroscopy for diabetes screening. J. Diabetes Sci. Technol. 2013, 7, 1001-1004. [CrossRef]

20. Olson, B.P.; Matter, N.I.; Ediger, M.N.; Hull, E.L.; Maynard, J.D. Noninvasive skin fluorescence spectroscopy is comparable to hemoglobin A1c and fasting plasma glucose for detection of abnormal glucose tolerance. J. Diabetes Sci. Technol. 2013, 7, 990-1000. [CrossRef]

21. Purroy, F.; Sánchez, E.; Lecube, A.; Arqué, G.; Vicente-Pascual, M.; Mauri-Capdevila, G.; Torreguitart, N.; Hernández, M.; Barbé, F.; Fernández, E.; et al. Prevalence and Predictors of Cerebral Microangiopathy Determined by Pulsatility Index in an Asymptomatic Population from the ILERVAS Project. Front. Neurol. 2021, 12, 785640. [PubMed]

22. Meerwaldt, R.; Graaff, R.; Oomen, P.H.N.; Links, T.P.; Jager, J.J.; Alderson, N.L.; Thorpe, S.R.; Baynes, J.W.; Gans, R.O.B.; Smit, A.J. Simple non-invasive assessment of advanced glycation endproduct accumulation. Diabetologia 2004, 47, 1324-1330. [CrossRef] [PubMed]

23. Lacolley, P.; Regnault, V.; Laurent, S. Mechanisms of arterial stiffening: From mechanotransduction to epigenetics. Arterioscler. Thromb. Vasc. Biol. 2020, 40, 1055-1062. [CrossRef] [PubMed]

24. Perrone, A.; Giovino, A.; Benny, J.; Martinelli, F. Advanced Glycation End Products (AGEs): Biochemistry, Signaling, Analytical Methods, and Epigenetic Effects. Oxidative Med. Cell. Longev. 2020, 2020, 3818196. [CrossRef] [PubMed]

25. Meerwaldt, R.; Lutgers, H.L.; Links, T.P.; Graaff, R.; Baynes, J.W.; Gans, R.O.; Smit, A.J. Skin autofluorescence is a strong predictor of cardiac mortality in diabetes. Diabetes Care 2007, 30, 107-112. [CrossRef]

26. Vlassara, H.; Uribarri, J. Advanced glycation end products (AGE) and diabetes: Cause, effect, or both? Curr. Diabetes Rep. 2014, 14, 453. [CrossRef]

27. Nowotny, K.; Jung, T.; Höhn, A.; Weber, D.; Grune, T. Advanced glycation end products and oxidative stress in type 2 diabetes mellitus. Biomolecules 2015, 16, 194-222. [CrossRef]

28. Lutgers, H.L.; Graaff, R.; Links, T.P.; Ubink-Veltmaat, L.J.; Bilo, H.J.; Gans, R.O.; Smit, A.J. Skin autofluorescence as a noninvasive marker of vascular damage in patients with type 2 diabetes. Diabetes Care 2006, 29, 2654-2659. [CrossRef]

29. Sánchez, E.; Betriu, À.; Yeramian, A.; Fernández, E.; Purroy, F.; Sánchez-de-la-Torre, M.; Pamplona, R.; Miquel, E.; Kerkeni, M.; Hernández, C.; et al. Skin Autofluorescence Measurement in Subclinical Atheromatous Disease: Results from the ILERVAS Project. J. Atheroscler. Thromb. 2019, 26, 879-889. [CrossRef]

30. van Waateringe, R.P.; Fokkens, B.T.; Slagter, S.N.; van der Klauw, M.M.; van Vliet-Ostaptchouk, J.V.; Graaff, R.; Paterson, A.D.; Smit, A.J.; Lutgers, H.L.; Wolffenbuttel, B.H.R. Skin autofluorescence predicts incident type 2 diabetes, cardiovascular disease and mortality in the general population. Diabetologia 2019, 62, 269-280. [CrossRef]

31. Rajaobelina, K.; Cougnard-Gregoire, A.; Delcourt, C.; Gin, H.; Barberger-Gateau, P.; Rigalleau, V. Autofluorescence of Skin Advanced Glycation End Products: Marker of Metabolic Memory in Elderly Population. J. Gerontol. Ser. A Biomed. Sci. Med. Sci. 2015, 70, 841-846. [CrossRef] [PubMed] 